\title{
Diurnal Salivary Cortisol following Mindfulness-Based Cognitive Therapy (MBCT) in Women with Breast Cancer: A pilot Study
}

\author{
Mohammad Taghi Badeleh (PhD) \\ Department of Psychology, \\ Kharazmi University, Tehran, Iran \\ Robabeh Noori (PhD) \\ Department of Psychology, \\ Kharazmi University, Tehran, Iran \\ Alireza Moradi (PhD) \\ Department of Psychology, \\ Kharazmi University, Tehran, Iran \\ Corresponding author: Robabeh \\ Noori \\ Tel: +989113711903 \\ Email: rynoury@yahoo.com \\ Address: Psychology Department, \\ Kharazmi University, Tehran, Iran \\ Received : 10 Apr 2018 \\ Revised: 04 Jun 2018 \\ Accepted: 30 Jun 2018
}

\begin{abstract}
Background and Objectives: Cancer and its conventional treatments may bring about some psychological and health-related symptoms leading to unstable cortisol level. Since evidence has shown that mindfulness based approaches can be helpful to reduce cortisol level, we aimed at investigating the efficacy of mindfulness-based cognitive therapy (MBCT) on decreasing salivary cortisol level in the women with breast cancer.
\end{abstract}

Methods: in this one- group pretest posttest study, the women with early stage breast cancer were assessed to select eligible ones $(\mathrm{N}=15)$. They were first trained for collecting salivary cortisol sample (before, after and follow-up) and then were asked to participate in MBCT, an eight-week session approach. Participants provided salivary samples in the morning (7: 30- 8:30), afternoon (1-3) and night (9-11). To measure cortisol, Cortisol Enzyme Immunoassay Kit was used. Analysis was performed on the data of 12 participants remained, using repeated measurement.

Results: the results of repeated measure ANOVA revealed that cortisol level of posttest (after 8-weeks of MBCT and follow-up period) in comparison with pretest was significantly reduced for morning and afternoon cortisol while it was not true for night cortisol. The post-treatment cortisol was not significantly correlated with age groups, education levels and socio-economic status using repeated measure ANCOVA.

Conclusion: Given that the women with breast cancer are at the high risk of psychological distresses and unstable cortisol level, we recommend mindfulness-based approaches esp. MBCT to health providers specifically those working with breast cancer patients.

Keywords: Mindfulness, MBCT, Cortisol, Salivary Cortisol, Breast cancer.

This paper should be cited as: Badeleh MT, Noori M, Sohrabi R, Moradi A[Diurnal Salivary Cortisol following Mindfulness-Based Cognitive Therapy (MBCT) in Women with Breast Cancer: A pilot Study]. mljgoums. 2018; 12(5):16-22 


\section{INTRODUCTION}

It is estimated that cancer is one of the leading causes of death. About 14 million new cancer diagnoses and 8.8 million cancerrelated deaths were reported, worldwide and this estimate has increased up to 22 million by two next decades (1). In Iran, cancer is the third cause of mortality following coronary heart disease and traffic accident, according to reports of the Iranian Ministry of Health and Medical Education (2). Breast cancer is the most frequently diagnosed cancer in women worldwide. The yearly incidence is 1.7 million and the five-year prevalence is 6.2 million, it is said that both are increasing (1).

Following medical and technological advances, the mortality rate has steadily declined and 5-year survival rate has progressively improved (3). By 2026, over 20 million cancer survivors will be alive in the United States alone (4). By and large, this increased chance of survival has given rise to some physical, financial, familial and occupational predicaments (5). In addition, based on Kenyon, Mayer, \& Owens, the survivors may suffer from some psychological symptoms including fear of recurrence anxiety, poor body image, cognitive impairment, sexual dysfunction, depression, and decreased life satisfaction (6) that lead to overactivity of stress response system- known as the HPA axis. Consequently, the physical and psychological stressors that women survivors of breast cancer encounter would be paramount importance to health clinicians.

As Patricia Gaete emphasized, both internal physiological stressors (cytokines, hypoxia, macromolecules, etc.) and external stressors (feelings of anxiety, fear, etc.) have influence on Hypothalamus-Pituitary-Adrenal (HPA) Axis and produce cortisol in excess (7). Shortly after perceiving a stressor, the corticotrophin-releasing hormone $(\mathrm{CRH})$ is secreted from the paraventricular nucleus in the hypothalamus. Then, $\mathrm{CRH}$ goes to pituitary gland and releases adrenocorticotropin hormone (ACTH) into the blood stream. ACTH binds to receptors in the adrenal cortex that stimulate secretion of cortisol. Most optimally, this stress response system regulates stress (8) and the appropriate functioning of this system is vital for physical and psychological well-being. However, although a short-term increase of cortisol can be adjustable, excessive or prolonged cortisol secretion may have debilitating effects, both physically and psychologically (9). Typically, cortisol level is at its peak level before awakening and declines incrementally over the rest of the day. In addition, studies consistently report a high correlation between serum and salivary cortisol, indicating salivary cortisol level reliably estimate serum cortisol level (10). Cortisol in patients with breast cancer was reported high compared to healthy people (11), indicating a maladaptive psychobiological stress response of the HPA axis (12). In most of breast cancer cases, psychological distresses persist for a long period of time implying the presence of an everlasting stress. They may be due to the uncertainty of their future, the unpredictability of the cancer, disability, financial difficulties, changing family situation and the problems at work. Moreover, physical appearance after mastectomy, hair loss from chemotherapy, and possible skin changes from radiation therapy are the other concerns that may lead to stress in breast cancer patients (5). When the stress becomes chronic and the HPA Axis is overactive, the chemicals like cortisol continue to be produced in excess, and this often leads to some health and psychological problems needing timely help (7). Besides, the increased cortisol level has to do with the reduction in the thickness of prefrontal cortex (13), suggesting the relation between chronic cancer-related stress and neuro- cognitive abilities. Hence, the stress-related effects emphasize on the need for psychological interventions. To mitigate heath and psychological-related consequences of cancer, patients may be unwilling to use conventional interventions. Instead, they resort to nonpharmacological approaches such as relaxation, coaching, cognitive behavioral therapy (CBT) self-delivered acupuncture and mindfulness (14) to make better off their capability for self-management. To cope with stress and likely modulate the psychobiological impacts of stress and cancer treatment, research shows that an almost stable coping resource with breast cancer is the patient's ability to accept the disease as a part of life. It means that the higher the acceptance, the lower psychological distresses (15). One of the acceptance-based approaches is mindfulness (a moment-to-moment awareness) focusing on non-judgment and accepting 
attitude to whatever arises. The most commonly used mindfulness-based interventions (MBI) are mindfulness-based stress reduction (MBSR) and mindfulnessbased cognitive therapy (MBCT) (16).

The mechanism of mindfulness- based interventions (MBIs) for different cancer patients has been clarified in an overview published in 2015 that indicated small to moderate effects on various psychosocial outcomes such as depression, anxiety and stress (17). In breast cancer patients, the effectiveness might be attributed to decreased reactivity, reduced catastrophizing, and increased self-kindness (18). Throughout treatment of breast cancer, the responses that are related to emotional coping remain stable, while those associated with cognitive coping are extremely variable (19). Moreover, owing to cancer or its treatment, some unhelpful cognition such as unwanted thoughts, images, or memories may occur in cancer survivors even several years following successful treatment. Some cancer patients become depressed, even with the thoughts of death and suicide (20).

The aforementioned cognition is accompanied by more robust physiological stress responses and slower recovery of cortisol levels following a stressor (21). This reveals the imperative role of the interventions that signify the role of cognition in the health of women who suffer from breast cancer. Of several approaches have been presented to date, Mindfulness Based Cognitive Therapy (MBCT) is the one that, in addition to mindfulness techniques consisting of breath awareness, body awareness and hatha yoga, emphasizes more on cognitive processes and rumination related to relapse in depression (16).

Mindfulness-based programs have been shown to be particularly effective in not only reducing distress and improving quality of life but also in regulating HPA axis function, restoring normal diurnal cortisol rhythms in patients with cancer (22).

Only a small number of studies have investigated the relationship between mindfulness-based interventions and cortisol in breast cancer patients; therefore, we aimed at evaluating the effect of MBCT on diurnal salivary cortisol in the patients suffering from breast cancer.

\section{MATERIAL AND METHODS}

The research protocol was approved by Health Psychology Department of Tehran Kharazmi University with the code of 4012999. Furthermore, all participants were asked to sign an informed consent and they have been assured of complete confidentiality for both their demographic and clinical information.

A group of breast cancer women was assessed in some oncology clinics of Gorgan city, Iran, in 2016-2017 to select eligible participants.

Age of between 20 and 59 years, stage I and II of breast cancer within one year, ability to attend the program, pledge to participate in all sessions of MBCT, ability to read and write in Farsi language, no experience of yoga or meditation courses in the past six months and signing of informed consent.

History of bipolar disorder, major depressive, psychotic disorder, substance abuse and personality disorder within past six months, based on Structured Clinical Interview for Diagnostic and Statistical Manual of Mental Disorders IV; certain diseases, such as Addison's disease and Cushing's disease, which affect the amount of cortisol; certain drugs that affect cortisol levels, such as drugs containing estrogen, synthetic glucocorticoids, drugs containing androgens and phenytoin; any other health conditions that would interfere with the practices of MBCT sessions. This within-subjects design (repeated measure design) was conducted on 15 eligible women with breast cancer selected amongst 238 women referred to oncology clinics. The participants $(n=15)$ were first invited to attend an orientation session in which they were asked to sign informed consent and were given the required instructions about collecting saliva samples to measure their awakening, afternoon and night cortisol levels. After obtaining the samples, they participated in 8week mindfulness-based cognitive behavioral therapy (MBCT) conducted by a licensed and trained therapist. Following the end of 8-week period and one month later, the participants who remained in the trial took salivary samples again to test cortisol level. At the end, each participant was given a gift for her participation in the study. For salivary cortisol collection, the participants were given sterile sample bottles labeled with identity code, and they were trained how to collect the samples. Before obtaining, they were asked not to eat or 
brush their teeth for an hour. In addition, within an hour of providing salivary sample, they were told not to smoke, not to drink coffee and not to use any substances that increase saliva production, e.g. lemon juice. To rinse their mouth, they were instructed to drink a half glass of water prior to taking the samples. Participants provided salivary samples immediately upon Waking (7: 30$8: 30)$, after noon (1-3) and at night (9-11). To facilitate cortisol collection, the women were also sent text-based reminder or they were contacted by phone. After obtaining the samples, the women were asked to keep the bottles in the refrigerator with the cap on until next morning to be transported by research assistant to the lab on ice. The samples were frozen at or below $-20^{\circ} \mathrm{C}$ to be evaluated later for cortisol level by Cortisol Enzyme Immunoassay Kit (Diametra, ( ${ }^{\circledR}$ Italy). To measure salivary cortisol, The Dia Metra ${ }^{\circledR}$ Cortisol Enzyme Immunoassay Kit was used. It is a Competitive immunoenzymatic colorimetric method for quantitative determination of Cortisol concentration in saliva. The intervention is mindfulness-based cognitive behavioral therapy (MBCT). It is a group-training program based on mindfulness and cognitive behavior therapy in which participants meet for $2-2.5 \mathrm{~h}$ per week over 8 weeks (23).

In addition to class exercises, the participants were encouraged to do home practice for at least $45 \mathrm{~min}$ per day to integrate the awareness skills into daily life. In order to address any potential concerns and ensure participants' adherence to treatment, the therapist tried to have every-other day phone contact. A clinical psychologist with sufficient experiences of mindfulness workshop and two well-trained women facilitators held the MBCT classes. The compliance with MBCT protocol was confirmed by the use of standardized manuals and specific checklists.

For data analysis, we used SPSS version 17.0. Demographic variables were described using frequency and descriptive statistics. Given the assumptions, repeated measures ANOVA was used to check the effect of MBCT on cortisol level of awakening, afternoon and night.

In addition, to see whether age, education and economic status were correlated with the effect of MBCT on cortisol levels, repeated measure ANCOVA was used.

\section{RESULTS}

Recruitment:The women referred to oncology clinics were assessed based on eligibility criteria and after that the appropriate ones $(n=15)$ were asked to participate in MBCT sessions.

In the course of the study, three of the participants could not continue due to personal reasons; therefore, statistical analyses was conducted on the data of 12 ones.

The studied women were mostly middle aged $(M=46.83 ; S D=7.93)$, high school educated and moderate level of socio-economic status. Pretest posttest comparison:Table 1 indicates that the salivary cortisol levels of awakening, afternoon and night are apparently different at baseline, after eight weeks of MBCT and onemonth follow up.

Repeated measure ANOVA was conducted to determine whether there was significant difference between cortisol level of posttest (after 8-weeks of MBCT and follow-up period) and pretest.

Given the assumptions including Mauchly's Test of Sphericity that was not statistically significant $(p \leq .05)$, the results revealed significant difference between repeated measures of awakening $[F(2,22)=5.11, p=$ $.015, \eta p 2=.32]$ and afternoon salivary cortisol $[F(2,22)=6.78, p=.005, \eta p 2=$ .38]. For night cortisol, because Mauchly's Test of Sphericity was statistically significant $(p \leq .05)$, the variances of the differences were not equal (i.e., Sphericity has been violated).

Thus, to combat the violation of the assumption of Sphericity, Greenhouse-Geisser correction was used and the results indicated that the MBCT effect on repeated measures of night salivary cortisol was not significant. [ $F$ $(2,22)=4.11, p=.062, \eta p 2=.27]$. Demographic variable were five levels of age group from 20 to 55, four levels of education including middle school, high school, associate and bachelor of Art, and three socioeconomic status levels including low, moderate and high level.

Based on repeated measure ANCOVA, the post-treatment scores were not significantly different in different age groups, education levels and socio-economic status levels. 
Table 1-Salivarv Cortisol level (ng/ml)

\begin{tabular}{lccc}
\hline & Baseline & $\begin{array}{l}\text { After8- } \\
\text { weeks }\end{array}$ & Follow-up \\
\hline Morning & $\mathbf{5 . 3 6} \pm 3.37$ & $4.59 \pm 2.73$ & $2.43 \pm 2.03$ \\
Afternoon & $2.47 \pm 1.69$ & $1.87 \pm 1.64$ & $0.84 \pm 1.46$ \\
Night & $1.91 \pm 2.27$ & $0.89 \pm 1.34$ & $0.76 \pm 1.35$ \\
\hline
\end{tabular}

\section{DISCUSSION}

Psycho-immunology research on the effect of mindfulness-based interventions is in its infancy and this study may be the first in Iran to demonstrate the effect of MBCT in decreasing cortisol in breast cancer survivors. Considering the data as preliminary evidence, this intervention was statistically effective in decreasing morning and afternoon cortisol level, while it was not the case for night cortisol. Additionally, the data revealed that the effect of MBCT on the levels of morning, afternoon and night salivary cortisol was not correlated with socio-demographic factors including age, education and socio-economic status. Nowadays the use of mindfulness-based interventions such as MBSR and MBCT is increasing. Recent studies show that MBCT can be helpful for psychological outcomes in some medical conditions, such as coronary heart disease (24) and cancer (25). It has also been clarified that mindfulness-based interventions are effective in supportive care of $\mathrm{BC}$ patients (26). In this case, we can mention the study of Lengacher et al. (27) for MBSR and the research of Johannsen et al. (28) for MBCT in breast cancer patients for alleviating some psychological variables such as stress. In addition to previous studies that have been about mindfulness based interventions (MBIs) and psychological outcomes, recently, some research has been conducted to assess the possible impact of MBIs on neuroendocrine physiology, HPA axis , by measuring cortisol level. For the cases other than cancer, we can mention the study of Laurent et al who reported that mindfulness, during a conflict discussion, could reduce the effects of negative partner behaviors on neuroendocrine stress responses (29). For the people suffering from cancer, some studies provide the evidence that cancer survivors with higher levels of MBIs may be buffered from deleterious changes in cortisol secretion (30). To the best of our knowledge, some research that has been carried out are associated with mindfulness based interventions and cortisol level $(31,32)$, and no MBCT trial exactly related to salivary cortisol of $\mathrm{BC}$ women has been reported.

In line with our study displaying the effect of MBCT on diurnal salivary cortisol, we can mention just the studies of Oken et al. (33), Daubenmier et al. (34) and Gex-Fabry et al. (35). These studies were carried out with the other participants other than $\mathrm{BC}$ patients including, caregivers, and overweight clients and depressed people, respectively. In the study of oken et al (33), the design was a pilot randomized trial to evaluate the effectiveness of a mindfulness meditation intervention adapted from MBCT that was conducted on 31 healthy adults providing care for a family member with dementia. Although, in contrast with our results, morning salivary cortisol in caregivers was not reduced significantly, there was significant correlation between mindfulness and self-rated mood and stress scores. In 2011, Daubenmier et al. (34) assessed the effect of Mindfulness-Based Eating Awareness Training, based on MBSR and MBCT, on cortisol level. In their study, 47 overweight and obese women with body mass index (BMI) between 25 and 40 were randomly assigned to a 4-month intervention or waitlist group. Even though mindfulness training demonstrated promise for improving eating patterns, neither group showed substantial changes in the diurnal pattern of cortisol. Their results were inconsistent with our results revealing MBCT can lessen morning salivary cortisol levels. Another study was carried out on 60 patients remitted from depression by Gex-Fabry et al. (35). They also found no significant effect of a MBCT on cortisol diurnal slope, which was not in agreement with our results for morning and afternoon cortisol.

The disparity between aforementioned studies and present study may be due to the kind of 
scales used to measure cortisol, the exact time of sampling, patients' condition, adherence to intervention, study limitations and large unexplained variability. In addition, in our study, we observe that the effect sizes for morning and afternoon cortisol are not high enough, and the change of night cortisol is not significant too. These shortcomings might be attributed to the points mentioned above and specifically to the limitations of our study that should be taken into account in future research.

This research has some strong points including the use of standard protocol (23) translated by a licensed therapist, performance by a skillful mindfulness therapist, every session check on the therapist's compliance with MBCT protocol and regular evaluation of participants' adherence to treatment. Despite the strengths, there are some shortcomings attributed mainly to absence of control group, small sample size and attrition reducing statistical power and generalization. Furthermore, most of the participants belong to middle-age group, moderate socio-economic status, high-school education level and self-motivated individuals. Additionally, awareness of being in a research group and the possibility of utilizing other palliative-care programs can be considered as confounding variables.

\section{REFERENCES}

1. Ferlay J, Soerjomataram I, Dikshit R, Eser S, Mathers $\mathrm{C}$, Rebelo $\mathrm{M}$, et al. Cancer incidence and mortality worldwide: sources, methods and major patterns in GLOBOCAN 2012. Int J Cancer. 2015; 136(5): E359E386. https://doi.org/10.1002/ijc.29210.

2. Naghavi M, Abolhassani F, Pourmalek F, Lakeh M, Jafari N, Vaseghi S, et al. The burden of disease and injury in Iran 2003. Popul Health Metr. 2009; 7: 9. https://doi.org/10.1186/1478-7954-7-9.

3. Demicheli R, Ambrogi F. Comparative benefit from small tumour size and adjuvant chemotherapy: clues for explaining breast cancer mortality decline. BMC Cancer. 2014;14: 702. https://doi.org/10.1186/1471-2407-14-702.

4. Miller KD, Siegel RL, Lin CC, Mariotto AB, Kramer $\mathrm{JL}$, Rowland $\mathrm{JH}$, et al. Cancer treatment and survivorship statistics, 2016. CA Cancer J Clin. 2016; 66: 271-289.

https://doi.org/10.3322/caac.21349.

5. American Cancer Society. Emotional Aspects of Breast Cancer Accessed 2/7/2014. https://my.clevelandclinic.org/health/diseases/8327-

breast-cancer-emotional-aspects.

6. Kenyon M, Mayer DK, Owens AK. Late and longterm effects of breast cancer treatment and surveillance management for the general practitioner. J Obstet Gynecol Neonatal Nurs. 2014; 43(3): 382-398. https://doi.org/10.1111/1552-6909.12300.

\section{CONCLUSION}

Overall, the results of present study conducted on Iranian samples indicate that mindfulness-based-cognitive therapy (MBCT) is a helpful approach for BC women undergoing oncologic treatments for example chemotherapy. This intervention could have significant effect on reducing psychological stress of BC patients and their cortisol levels. Based on the results, MBCT could not decrease night cortisol significantly compared to morning and afternoon cortisol. In order to clarify the effectiveness, further comprehensive research is needed to investigate the influence of MBCT, considering individual characteristics.

\section{ACKNOWLEDGMENTS}

The authors would like to thank psychology department of Tehran Kharazmi University for their steady counseling and to all participants who joined eagerly in this study. Furthermore, we are thankful to Mohamad Ali vakili, $\mathrm{PhD}$ of biostatistics, for his scientific and helpful comments, and we appreciate perfect contribution of Kavosh Laboratory in Gorgan.

\section{CONFLICT OF INTEREST}

All authors declare that we have no conflicts of interest.

7. Gaete HP. Hypothalamus-Pituitary-Adrenal Axis, chronic stress, hair cortisol, metabolic syndrome, and mindfulness. Psychoneuroendocrinology. 2016; 71: 28. https://doi.org/10.1016/j.psyneuen.2016.07.079.

8. Kudielka BM, Kirschbaum C. Sex differences in HPA axis responses to stress: a review. Biological psychology. 2005; 69(1): 113-132. https://doi.org/10.1016/j.biopsycho.2004.11.009.

9. Hannibal KE, Bishop MD. Chronic stress, cortisol dysfunction, and pain: a psychoneuroendocrine rationale for stress management in pain rehabilitation. Phys Ther. 2014; $94(12)$ : 1816-25. https://doi.org/10.2522/ptj.20130597.

10. Dorn LD, Kolko DJ, Susman EJ, Huang B, Stein H, Music E, et al. Salivary gonadal and adrenal hormone differences in boys and girls with and without disruptive behavior disorders: Contextual variants. Biological psychology. 2009; 81: 31-39. https://doi.org/10.1016/j.biopsycho.2009.01.004

11. McGregor BA, Antoni MH. Psychological intervention and health outcomes among women treated for breast cancer: a review of stress pathways and biological mediators. Brain Behav Immun. 2009; 23: 159-166. https://doi.org/10.1016/j.bbi.2008.08.002. 
12. McEwen BS. The neurobiology of stress: from serendipity to clinical relevance. Brain research. 2000; 886(1-2): $\quad 172-189 . \quad$ https://doi.org/10.1016/S00068993(00)02950-4.

13. Kremen WS, O'brien RC, Panizzon MS, PromWormley E, Eaves LJ, Eisen SA, et al. Salivary cortisol and prefrontal cortical thickness in middle-aged men: A twin study. Neuroimage. 2010; 53: 1093-1102. https://doi.org/10.1016/j.neuroimage.2010.02.026.

14. Rogers A, Kennedy A, Bower $\mathrm{P}$, et al. The United Kingdom Expert Patients Programme: results and implications from a national evaluation. Medical Journal of Australia. 2008; 189 (10 Suppl): S21-4.

15. Witek-Janusek L, Albuquerque K, Chroniak KR, Chroniak C, Durazo-Arvizu R, Mathews HL. Effect of mindfulness based stress reduction on immune function, quality of life and coping in women newly diagnosed with early stage breast cancer. Brain, behavior, and $\begin{array}{lll}\text { immunity. } & 2008 ; & 22:\end{array}$ https://doi.org/10.1016/j.bbi.2008.01.012.

16. Segal ZV, Williams JMG, Teasdale JD. Mindfulnessbased cognitive therapy for depression. Guilford Press, 2012.

17. Gotink RA, Chu P, Busschbach JJ, Benson H, Fricchione GL, Hunink MM. Standardised mindfulnessbased interventions in healthcare: an overview of systematic reviews and meta-analyses of RCTs. PloS one. 2015; 10(4): e0124344.

https://doi.org/10.1371/journal.pone.0124344.

18. Johannsen M, O'connor M, O'toole MS, Jensen AB, Zachariae R. Mindfulness-based Cognitive Therapy and Persistent Pain in Women Treated for Primary Breast Cancer: Exploring Possible Statistical Mediators Results From a Randomized Controlled Trial. Clin J Pain. 2018; 34(1): 59-67.

19. Hervatin R, Sperlich S, Koch-Giesselmann H, Geyer S. Variability and stability of coping in women with breast cancer. Supportive Care in Cancer. 2012; 20: 2277-2285.

https://doi.org/10.1007/s00520-011-1334-2.

20. Bleiker EM, Pouwer F, Van Der Ploeg HM, Leer JWH, Adèr HJ. Psychological distress two years after diagnosis of breast cancer: frequency and prediction. Patient education and counseling. 2000; 40(3): 209-217. https://doi.org/10.1016/S0738-3991(99)00085-3.

21. Zoccola PM, Dickerson SS. Assessing the relationship between rumination and cortisol: A review. Journal of psychosomatic research. 2012; 73(1): 1-9. https://doi.org/10.1016/j.jpsychores.2012.03.007.

22. Smith JE, Richardson J, Hoffman C, Pilkington K. Mindfulness-Based Stress Reduction as supportive therapy in cancer care: systematic review. J Adv Nurs. 2005; 52(3): 315-327.

https://doi.org/10.1111/j.1365-2648.2005.03592.x.

23. Crane R. Mindfulness-based cognitive therapy: Distinctive features. Taylor \& Francis. 2009.

24. O'Doherty V, Carr A, McGrann A, O'Neill JO, Dinan $\mathrm{S}$, Graham I, et al. A controlled evaluation of mindfulness-based cognitive therapy for patients with coronary heart disease and depression. Mindfulness. 2015; 6(3): 405-16.

https://doi.org/10.1007/s12671-013-0272-0.
25. Kingston T, Collier S, Hevey D, McCormick M, Besani C, Cooney J, et al. Mindfulness-based cognitive therapy for psycho-oncology patients: an exploratory study. Irish Journal of Psychological Medicine. 2015; 32(3): 265-74. https://doi.org/10.1017/ipm.2014.81.

26. Huang J, Shi L. The effectiveness of mindfulnessbased stress reduction (MBSR) for survivors of breast cancer: study protocol for a randomized controlled trial. Trials. 2016; 17(1): 209. doi: 10.1186/s13063-016-1335$\mathrm{z}$.

27. Lengacher CA, Reich RR, Paterson CL, Ramesar S, Park JY, Alinat C, et al. Examination of Broad Symptom Improvement Resulting From Mindfulness-Based Stress Reduction in Breast Cancer Survivors: A Randomized Controlled Trial. J Clin Oncol. 2016; 34(24): 2827-34. https://doi.org/10.1200/JCO.2015.65.7874.

28. Johannsen M, O'Connor M, O'Toole MS, Jensen AB, Højris I, Zachariae R. Efficacy of mindfulness-based cognitive therapy on late post-treatment pain in women treated for primary breast cancer: a randomized controlled trial. Journal of clinical oncology. 2016; 34(28): 3390-9. https://doi.org/10.1200/JCO.2015.65.0770

29. Laurent HK, Hertz R, Nelson B, Laurent SM. Mindfulness during romantic conflict moderates the impact of negative partner behaviors on cortisol responses. Horm Behav. 2016; 79: 45-51. DOI:10.1016/j.yhbeh.2016.01.005.

30. Black DS, Peng C, Sleight AG, Nguyen N, Lenz HJ, Figueiredo JC. Mindfulness practice reduces cortisol blunting during chemotherapy: A randomized controlled study of colorectal cancer patients. Cancer. 2017; 123(16): 3088-3096.https://doi.org/10.1002/cncr.30698.

31. Sanada K, Montero-Marin J, Alda Díez M, SalasValero M, Pérez-Yus MC, Morillo $\mathrm{H}$, et al. Effects of Mindfulness-based interventions on salivary cortisol in healthy adults: a meta-analytical review. Frontiers in physiology. 2016; 7: 471. eCollection 2016. https://doi.org/10.3389/fphys.2016.00471.

32. Matousek RH, Pruessner JC, Dobkin PL. Changes in the cortisol awakening response (CAR) following participation in mindfulness-based stress reduction in women who completed treatment for breast cancer. Complement Ther Clin Pract. 2011; 17(2): 65-70. https://doi.org/10.1016/j.ctcp.2010.10.005.

33. Oken BS, Fonareva I, Haas M, et al. Pilot controlled trial of mindfulness meditation and education for dementia caregivers. The Journal of Alternative and Complementary Medicine. 2010; 16(10): 1031-1038. https://doi.org/10.1089/acm.2009.0733.

34. Daubenmier J, Kristeller J, Hecht FM, et al. Mindfulness intervention for stress eating to reduce cortisol and abdominal fat among overweight and obese women: an exploratory randomized controlled study. Journal of obesity. 2011; 2011.

35. Gex-Fabry M, Jermann F, Kosel M, Rossier MF, Van der Linden M, Bertschy G, et al. Salivary cortisol profiles in patients remitted from recurrent depression: One-year follow-up of a mindfulness-based cognitive therapy trial. J Psychiatr Res. 2012; 46(1): 80-86. https://doi.org/10.1016/j.jpsychires.2011.09.011. 